
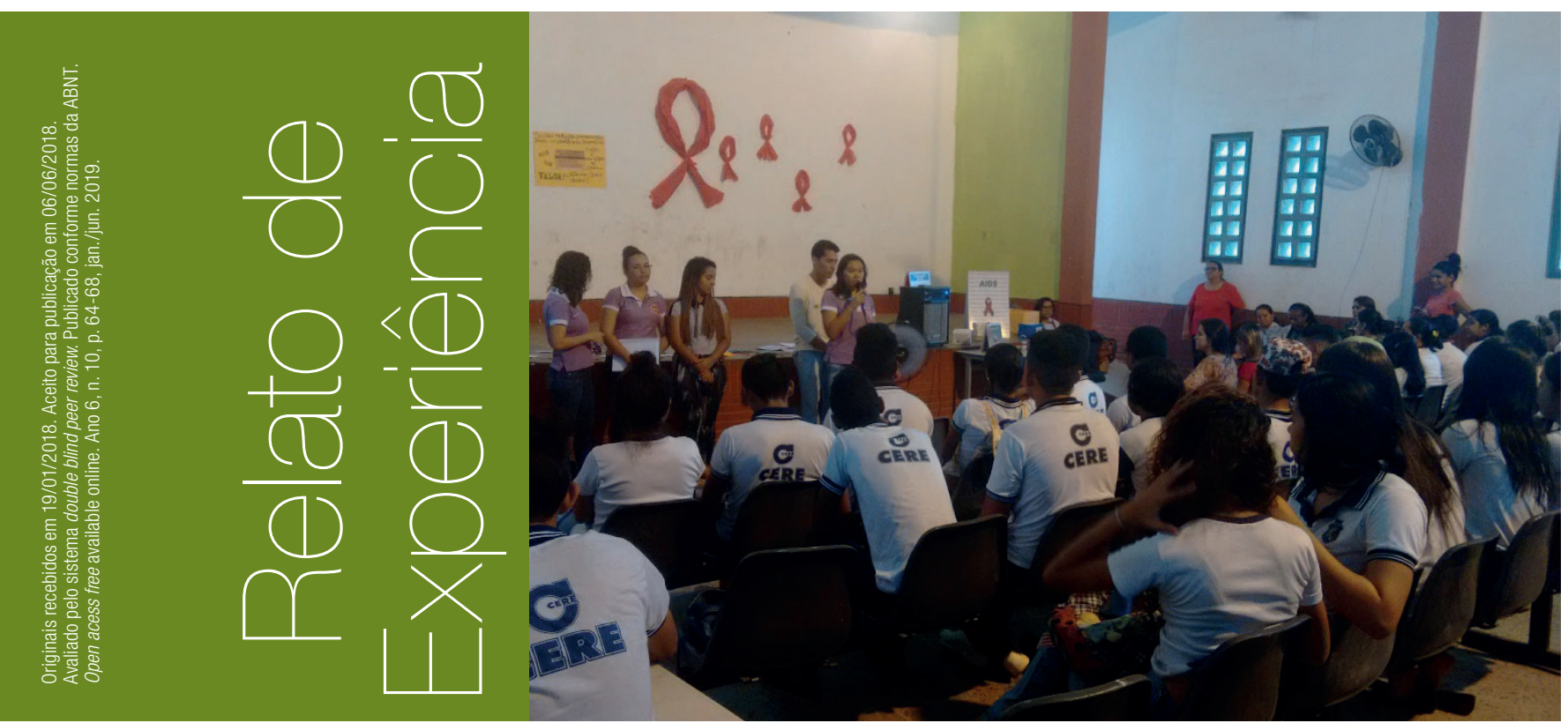

\title{
Promoção da saúde do adolescente: experiências acadêmicas em uma liga de extensão
}

\author{
Maria Aline Moreira Ximenes - aline.ximenes11@hotmail.com \\ Maria Girlane Sousa Albuquerque Brandão - \\ girlane.albuquerque@yahoo.com.br ${ }^{2}$ \\ Sibele Pontes Rocha - sibelepontes@hotmail.com³
}

\section{RESUMO}

Este relato apresenta a experiência de uma liga de extensão de promoção à saúde do adolescente. Foram realizadas mais de 100 ações de extensão durante o período de abril de 2016 a dezembro de 2017, o que viabilizou a abordagem de cerca de 1.200 adolescentes em escolas, quadras, praças, unidades de saúde, Centros de Referências e Assistência Psicossocial de Sobral, Ceará, com foco na elaboração de ações educativas, disseminação de conhecimentos e promoção da saúde do público adolescente.

\section{PALAVRAS-CHAVE}

Promoção da Saúde. Tecnologia Educacional. Adolescência. Enfermagem.

\footnotetext{
1 Acadêmica de Enfermagem pela Universidade Estadual Vale do Acaraú- UVA, Membro do Grupo de Estudos de Cuidados Clínicos de Enfermagem na Saúde do Adulto, Monitora Voluntária do Módulo de Vida Adulta e Bolsista de Iniciação Científica PROVIC-UVA

2 Científica PROVIC-UVA Graduanda em Enfermagem pela Universidade Estadual Vale do Acaraú - UVA. Bolsista de Iniciação Científica. Participou da Liga de Promoção à Saúde do Adolescente. Integra a organização internacional ENACTUS. Bolsista de Iniciação Científica Voluntária (PROVIC/UVA).
}

3 Integrante do grupo de estudos e pesquisa em Cuidados e Enfermagem na Saúde do Adulto. Enfermeira. Mestranda em Saúde da Família pela Universidade Federal do Ceará. 


\section{ABSTRACT}

This report presents the experience of an extension league for adolescent health promotion. More than 100 extension actions were carried out from April 2016 to December 2017, which enabled the approach of about 1,200 adolescents in schools, blocks, squares, health units, Centers of Reference and Psychosocial Assistance of Sobral, Ceará, focusing on the elaboration of educational actions, dissemination of knowledge and health promotion of the adolescent public.

\section{KEYWORDS}

Health Promotion. Educational Technology. Adolescence. Nursing.

\section{Relato de experiência}

A adolescência é um período de mudanças entre a infância e a idade adulta marcada por transformações nos âmbitos físico e psicossocial, relacionadas principalmente a busca da autoafirmação de identidade, aceleração do desenvolvimento intelectual e anatômico e evolução da sexualidade (FELIPE et al., 2011).

0 exercício da sexualidade na adolescência pode se tornar um problema, devido à falta de informação em saúde sexual, deficiências na comunicação entre os familiares, influência de tabus e crenças e reprodução de comportamentos de amigos do mesmo círculo social. Essas influências sociais podem favorecer a adoção de comportamentos sexuais de risco, como 0 não uso, ou uso incorreto, de preservativos, que aumentam a vulnerabilidade para a gravidez inoportuna e para as infecções sexualmente transmissíveis (IST), com destaque para o vírus da imunodeficiência humana (HIV) e da síndrome da imunodeficiência adquirida (Aids) (FREITAS, 2017).

A estratégia básica para 0 controle da transmissão das IST/HIV e Aids é a prevenção, por meio de atividades educativas que focalizem a vulnerabilidade inerente a uma relação sexual desprotegida, a mudança no comportamento e a adoção dos preservativos (BRASIL, 2006).

A Liga de Promoção a Saúde do Adolescente (LIPSA) é uma liga de extensão acadêmica vinculada ao Curso de Enfermagem da Universidade Estadual Vale do Acaraú (UVA), em Sobral - CE. 0 objetivo da LIPSA é contribuir na formação de profissionais capacitados para atuar na atenção à saúde do adolescente, com foco na educação como instrumento de sensibilização, mudança de comportamentos de risco e o protagonismo juvenil, tornando o jovem agente de sua própria saúde e multiplicador de estilos de vida saudáveis.

Este artigo tem como finalidade abordar experiências de acadêmicas a partir das práticas extensionistas da LIPSA. Expõe-se a proposta das atividades com base em tecnologias educativas e os resultados obtidos diante das ações realizadas com os adolescentes em diversos ambientes, como escolas, ruas e em grupos de jovens da comunidade.

As ações e atividades de extensão desenvolvidas pela LIPSA foram realizadas por discentes de enfermagem da UVA, apoiados por diversos dispositivos, como a Estratégia Saúde da Família (ESF) e o Programa Saúde na Escola (PSE), vinculados à Secretaria de Saúde do Município de Sobral; o Projeto Flor do Mandacaru criado na cidade para atuar na assistência à saúde sexual e reprodutiva do adolescente; bem como a Rede de Educação de Sobral e o corpo docente da UVA.

As ações de extensão foram desenvolvidas de acordo com a demanda do município, o que viabilizou a abordagem de cerca de 1.200 adolescentes de vários bairros da cidade. Os espaços articulados para 0 desenvolvimento das atividades compreenderam espaços sociais representados por escolas, quadras, praças, unidades de saúde, Centros de Referências e Assistência Psicossocial (CREAS) e diversos espaços de encontros da comunidade, sendo realizadas mais de 100 ações no período de abril de 2016 a dezembro de 2017. Entre as 
atividades desenvolvidas com os adolescentes, destacam-se os jogos educativos, que foram muito utilizados, visto que atraíam a participação e 0 interesse dos jovens para refletir sobre temáticas recorrentes e causadoras de problemas nesta fase da vida, tais como: gravidez na adolescência, IST, o uso de drogas, acidentes de trânsito, a violência e o suicídio.

Figura 1: Ação de extensão na Semana Municipal do Adolescente na Rua do Bolevard do Arco, Sobral, Ceará, dezembro/2016.

Fonte: Acervo da pesquisa

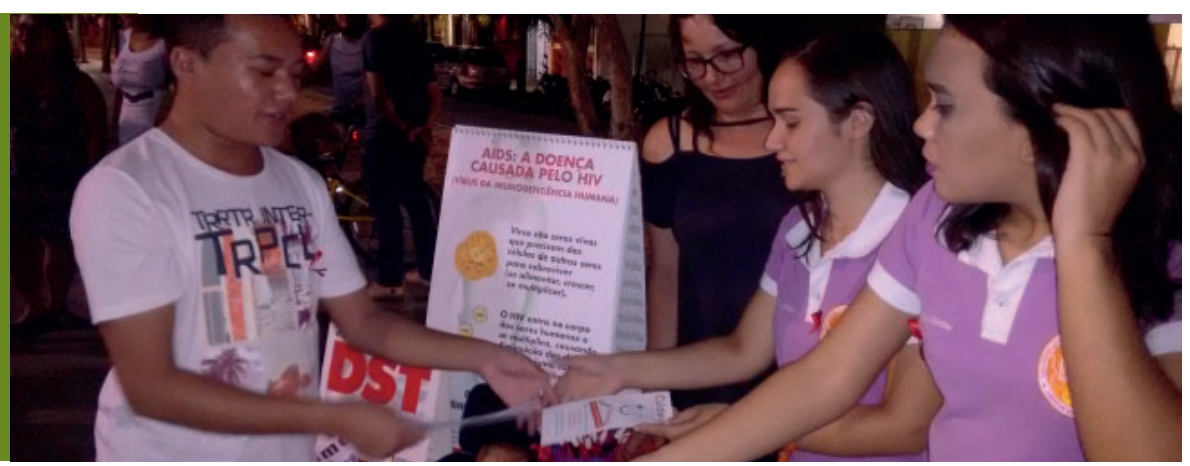

Figura 2: Ação de extensão no VII Encontro de Extensão da Universidade Estadual Vale do Acaraú, Sobral, Ceará, outubro/2016.

Fonte: Acervo da pesquisa

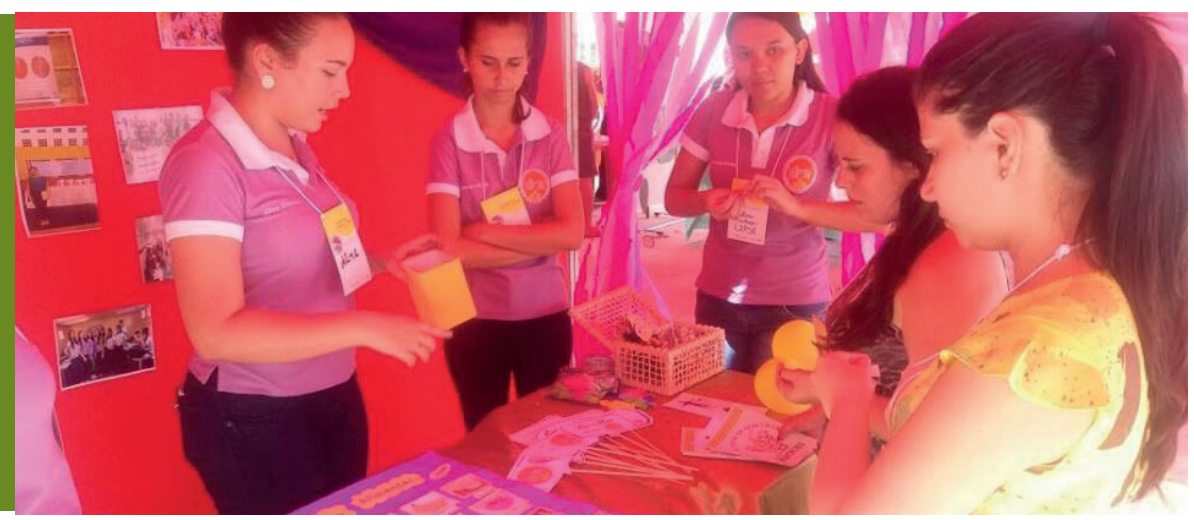

É importante dar ao jovem a oportunidade de ele ser autor da própria história, a partir do desenvolvimento do protagonismo juvenil, engajando-o em projetos que ele mesmo crie, assuma e administre. Para incentivar os adolescentes e jovens a participar das atividades de promoção de saúde, é interessante a organização de gincanas, competições e jogos (BRASIL, 2017).

Dentre os jogos mais utilizados destacam-se: 0 tabuleiro alimentar, o qual tem como objetivo promover hábitos alimentares saudáveis e a prática de exercícios; 0 jogo da velha sobre diretos sexuais e reprodutivos; o jogo mitos ou verdades sobre IST; 0 tabuleiro da educação sexual; 0 jogo das situações-problemas sobre álcool e drogas, dentre outros.

A escola foi o principal campo de atuação da LIPSA, onde foi possível reunir jovens da mesma faixa etária, com espaço adequado e apoio de professores. No entanto, algumas ações foram realizadas nas ruas durante eventos municipais, abordando temas de saúde com jovens protagonistas, os quais participavam de grupos da comunidade. Além disso, foram realizadas oficinas com adolescentes de outras cidades na própria universidade por meio de uma mostra de projetos de extensão. Dessa forma, foi possível disseminar conhecimentos entre diversos atores, ultrapassando os limites da universidade e até mesmo de Sobral, visto que muitos professores e alunos referiram interesse em replicar os jogos apresentados nas escolas de seu município.

Diante das experiências obtidas nas ações, foi possível perceber o engajamento dos adolescentes e 0 interesse dos mesmos. Os encontros eram de fato espaços de promoção da saúde em sua forma ampliada, por meio do estabelecimento de vínculos, solução de dúvidas e favorecimento de momentos de diversão, cultura, lazer e troca de conhecimentos.

A LIPSA também tem impactos positivos nos acadêmicos de enfermagem, contribuindo para uma formação de qualidade, mais sensível aos anseios do público adolescente e jovem, o que também pode transformar a realidade atual dos serviços de saúde, preenchendo lacunas relacionais a atenção à saúde desse público. É importante haver mudanças na prática profissional, de forma a incorporar a assistência integral aos adolescentes em conjunto com as equipes e gestores, objetivando o estabelecimento da qualidade desta assistência (FERREIRA et al., 2016). 
Com relação às dificuldades encontradas, destaca-se a falta de apoio de alguns gestores na abertura de espaço para ações de promoção da saúde. No que diz respeito aos adolescentes, os principais desafios foram a resistência de alguns à participação nos momentos em grupo e na ausência de ações voltadas aos jovens não institucionalizados, que não frequentam a escola ou grupos da comunidade.
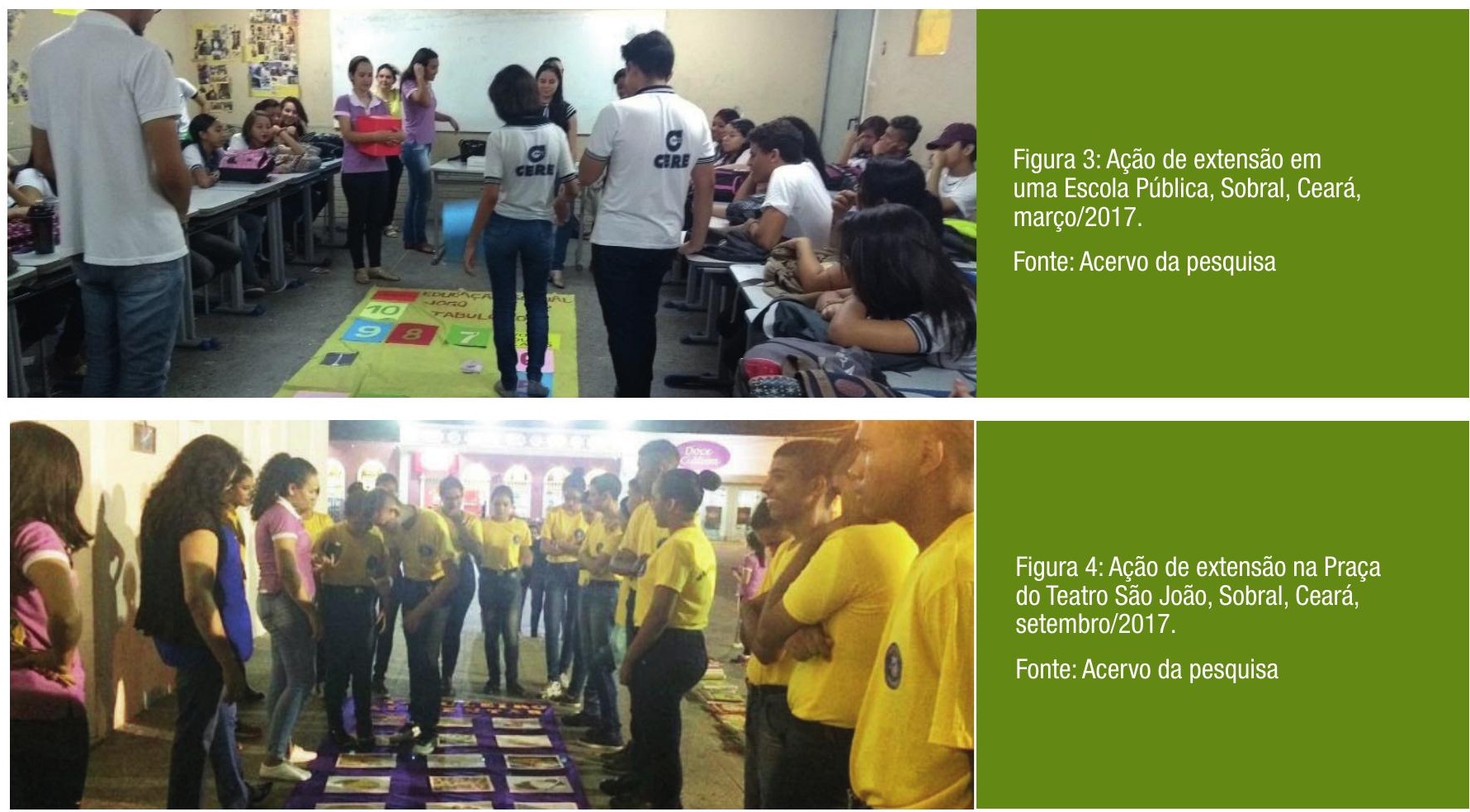

Figura 4: Ação de extensão na Praça do Teatro São João, Sobral, Ceará, setembro/2017.

Fonte: Acervo da pesquisa

Um fator muito importante em todas as ações promovidas pela LIPSA foi a avaliação dos adolescentes, sempre realizada ao final das atividades como forma de mensurar a efetividade das tecnologias utilizadas na transmissão de conhecimentos. Os jovens tinham a oportunidade de expressar livremente suas opiniões, que repercutiam nos encontros seguintes e no roteiro de atividades propostas. Os feedbacks foram positivos, principalmente em relação à metodologia utilizada, o que pôde ser percebido por meio das solicitações de retorno da Liga, pelos gestores dos espaços trabalhados e pelos próprios adolescentes.

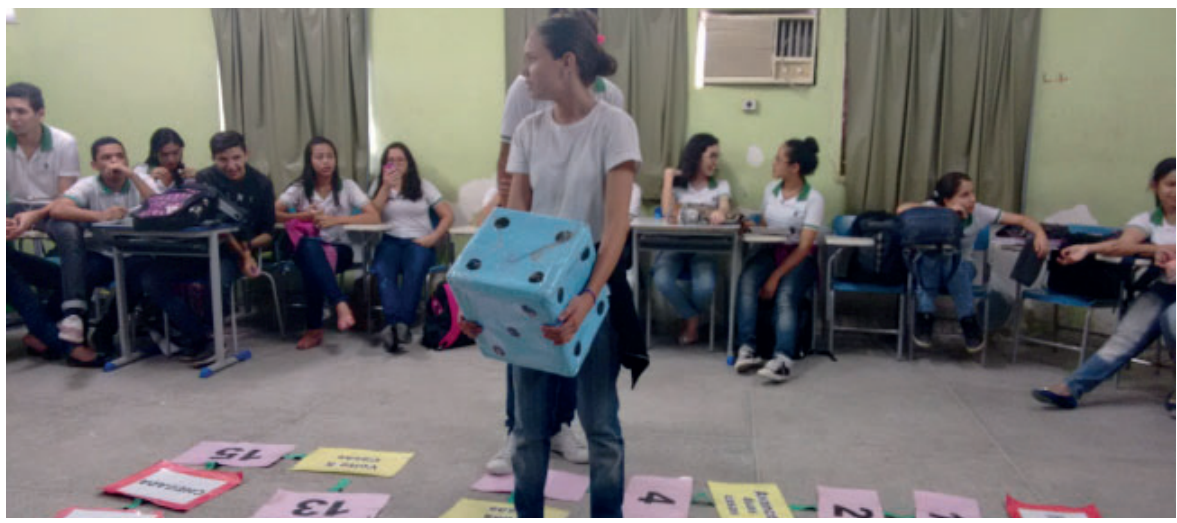

Figura 5: Ação de extensão em uma escola pública, Sobral, Ceará, dezembro /2016.

Fonte: Acervo da pesquisa

Dessa forma, diante das limitações vivenciadas e do feedback dos adolescentes a cada intervenção, pode-se sugerir que, em futuras práticas de promoção da saúde, sejam utilizados formulários padronizados para avaliação, como testes antes e após as ações, afim de analisar a efetividade do encontro, a aprendizagem e fixação dos conhecimentos. Além disso, ressalta-se a necessidade de inserir professores e gestores nas atividades propostas, assim como capacitar estes profissionais, para que reconheçam a importância de promover saúde nos diferentes espaços e que sejam aptos a realizarem as metodologias sugeridas pela LIPSA. 


\section{Referências}

BRASIL. Ministério da Saúde. Secretaria de Atenção à Saúde. Departamento de Ações Programáticas e Estratégicas. Proteger e cuidar da saúde de adolescentes na atenção básica. Brasília, 2017.

BRASIL. Ministério da Saúde. Secretaria de vigilância em saúde. Programa nacional de DST e Aids. Manual de bolso das doenças sexualmente transmissíveis. Brasília, DF; 2006.

FELIPE, A.B.O; ANDRADE, C.U.B; FÁBIO, S.T; ALCKMIN, B.A; ÁVILA, T.W.S. Análise do conhecimento do adolescente sobre os métodos anticoncepcionais. RevEnferm UFPE. 2011.

FERREIRA, I.S.V; SILVA, C.C; LUCINDO, M.D.F; NEGREIROS, R.V. Discutindo Saúde Sexual e Reprodutiva com Adolescentes do Ensino Fundamental: Relato de Experiência. Caminho Aberto - Revista de Extensão do IFSC, ano 3, nº 4, julho de 2016.

FREITAS, N.0; CARVALHO, K.E.G; ARAÚJO, E.C. Estratégia de educação em saúde para um grupo de adolescentes do Recife. Adolesc Saúde. 2017 